\title{
Structural features of mineral carriers of medicinal substances
}

\author{
Alexander Bondarev*, Elena Zhilyakova, Anastasia Malyutina, Larissa Kozubova, Natalia \\ Avtina, Elena Timoshenko, and Georgy Vasiliev \\ ${ }^{1}$ Belgorod State National Research University, 308015, Belgorod, Russia
}

\begin{abstract}
The aim of the work is to investigation of the structural features of mineral carriers of medicinal substances. Tasks: conduct electron microscopy and study the structural features of mineral sorbents; develop a classification of sorption interaction. The materials are Smectite Dioctahedral (registration certificate N 015155/01, France), Kaolin (state standard 19608-84, Russia), Montmorillonite Clay (technical specifications 9296-001-62646221-2012, Russia). The methods are scanning electron microscopy on a FEI Quanta 600 microscope with a low vacuum mode and an LFD detector. Results. Electron microscopy of objects was performed using segmentation of elements as subsystems, inside which the morphological description does not penetrate. The morphology of objects is studied. It is established that the studied substances are microstructural objects. Porosity was determined in samples of Smectite, Kaolin and Montmorillonite Clay. The classification of sorption interaction is developed. According to the presented classification, the materials under study are divided into two groups according to their porous characteristics: Group 1-sorption interaction in pores and by ion exchange (Smectite, Montmorillonite Clay); Group 2-sorption in secondary pores and by means of Oxygen and Hydroxyl centers (Kaolin).
\end{abstract}

Keywords: minerals, sorbents, sorption.

\section{Introduction}

Currently, mineral clays are widely used in medicine and pharmacy. Mineral clays can be used to create drug transport systems in which the sorption mechanism is used to bind, transport, and isolate the drug molecule. In this case, the sorbent acts as a carrier of the drug molecule with its subsequent delivery to the destination by desorption [1].

One of the ways to study the sorption-desorption processes in drug transport systems is to study the morphological structure of the sorption substance. Morphological structure refers to the aggregate characteristics of elements, including their size, shape, and spatial organization. The morphology of the elements can differ significantly depending on the composition of the material, its crystal structure and the method of preparation. Knowing the physical and chemical properties of the substance, in particular the surface morphology, it is possible to predict the sorption characteristics [2].

\footnotetext{
*Corresponding author: alexbond936@yandex.ru
} 


\section{Experimental}

The following materials were used in the study: Smectite Dioctahedral (registration certificate N 015155/01, France), Kaolin (state standard 19608-84, Russia), Montmorillonite Clay (technical specifications 9296-001-62646221-2012, Russia).

Scanning electron microscopy was used to study the morphology. The FEI Quanta 600 microscope with low vacuum mode and LFD detector uses the principle of scanning a thin beam of electrons over the sample surface. Each point of the sample is successively irradiated by a focused electron beam that moves along the surface under study, resulting in response signals of various physical nature (reflected and secondary electrons, Auger electrons, X-rays), which are used for synchronous image construction.

\section{Results and discussion}

The microscopy was carried out on the basis of the Center for Collective Use "Technologies and Materials" of the National Research University "BelSU". When describing the morphology of objects, the methods presented in [3] were used. Medical Clay is a medicine containing substances of mineral origin, subjected to primary processing in order to remove non-clay solids and water-soluble salts, which has adsorption activity and is intended for the manufacture of drugs with an adsorption effect.

To perform the analysis, we used the metric presented in the lower right corner of each micrograph, as well as segmentation of elements as subsystems, inside which the description does not penetrate.

The obtained Smectite micrographs are presented in fig. 1.
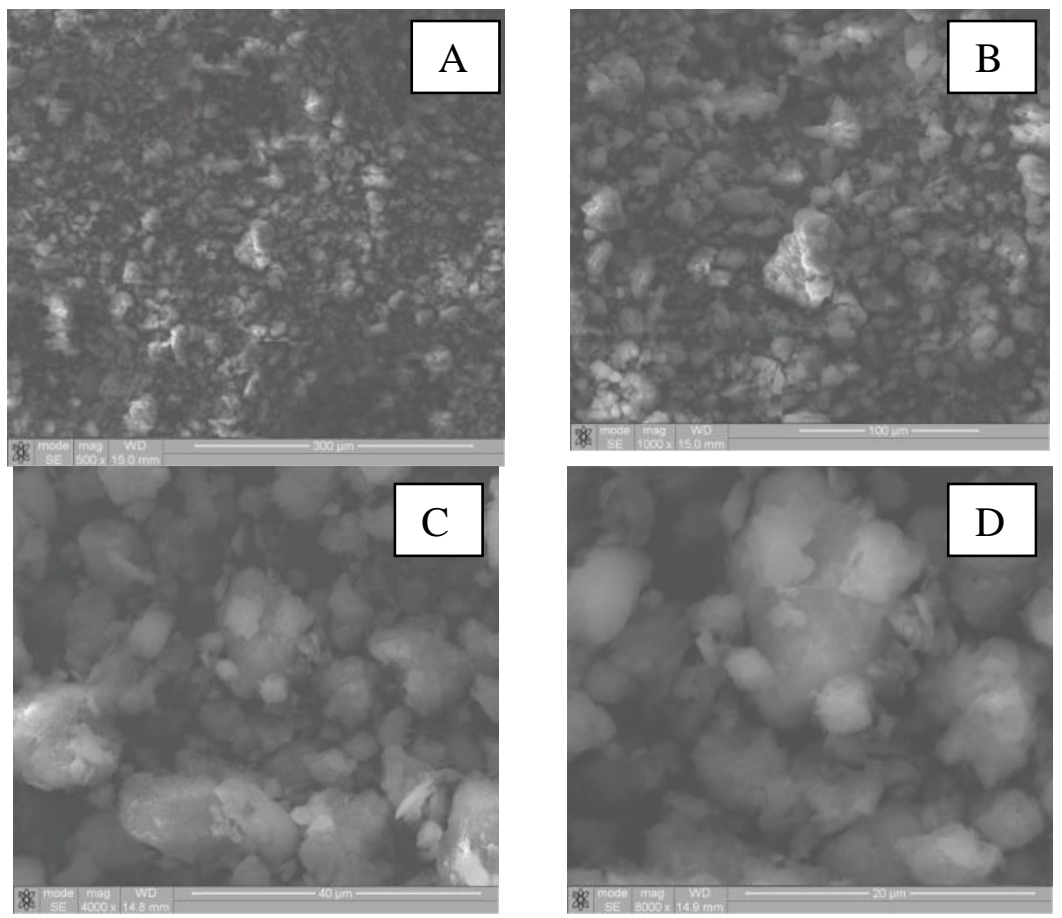

Fig. 1. Smectite micrographs. scale segment: a-300 microns, b-100 microns, c- 40 microns, d-20 microns 
Segmentation of elements is represented by two levels of organization: level 1 is micrographs a-b, the second level is c-d.

Smectite at the first level of organization is a cluster of round, oval, lamellar particles. The average particle size was 5-20 microns. The second level, when magnified by 4-8 thousand times, is represented by plate elements combined into round-shaped particlesglobules with a size of 1-20 microns. There are pores formed by the connection of the lamellar elements with each other.

Fig. 2 shows micrographs of Kaolin. Segmentation of elements is represented by two levels of organization: level 1 is micrographs $a-b$, the second level is c-d.
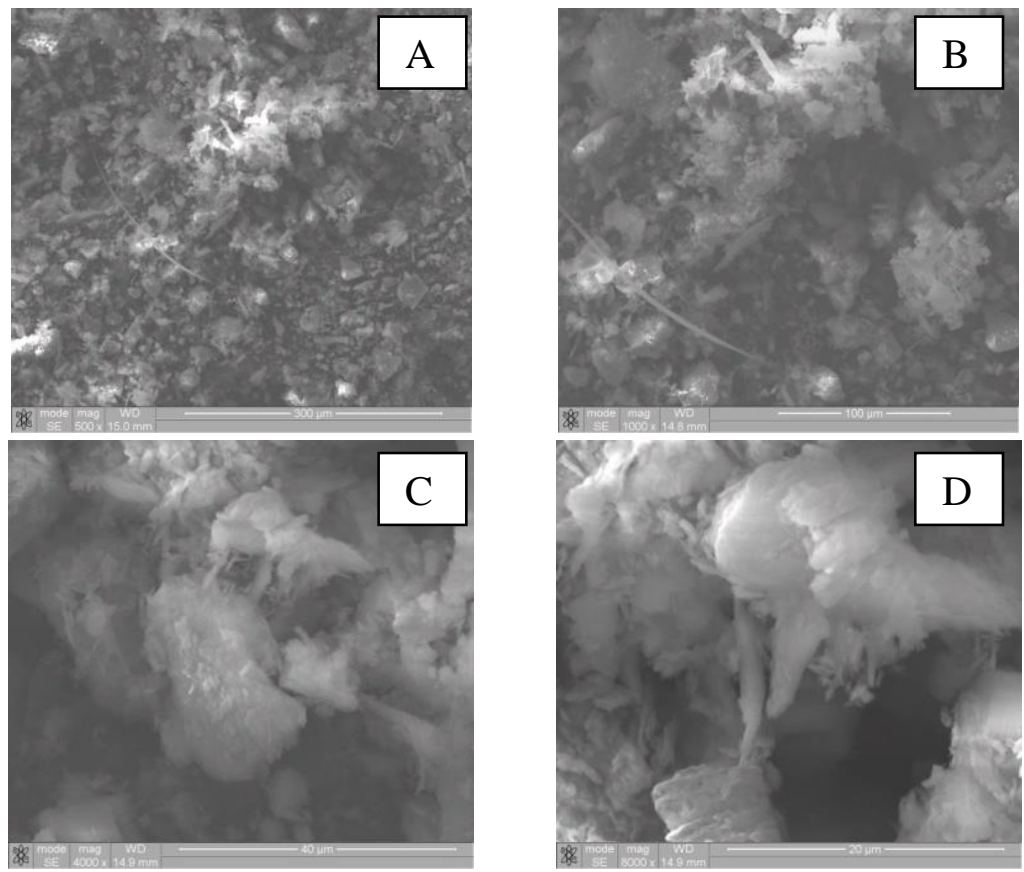

Fig. 2. Micrographs of kaolin. scale segment: a-300 microns, b-100 microns, c-40 microns, d-20 microns

At the first level of organization, Kaolin consists of irregularly shaped particles with a size of 5-20 microns, and elements with a size of up to 50 microns are found. The second level, with an increase of 4-8 thousand times, is represented by elements combined with each other in irregularly shaped particles of 1-20 microns in size with a developed rough surface. There are pores formed by the connection of the plate elements with each other.

Fig. 3 shows micrographs of Montmorillonite Clay. Segmentation of elements is represented by two levels of organization: level 1 is micrographs a-b, the second level is c-d.

Montmorillonite Clay at the first level of organization consists of equiaxed plate-shaped particles with an uneven surface, sharp edges, and cracks; the average particle size was 2-5 microns. There are aggregates with flake-like outlines, folded formations. At the second level of organization, at an increase of 4-8 thousand times, the lamellar particles are combined into larger elements. There are pores of various sizes formed by the connection of the plate elements with each other. 

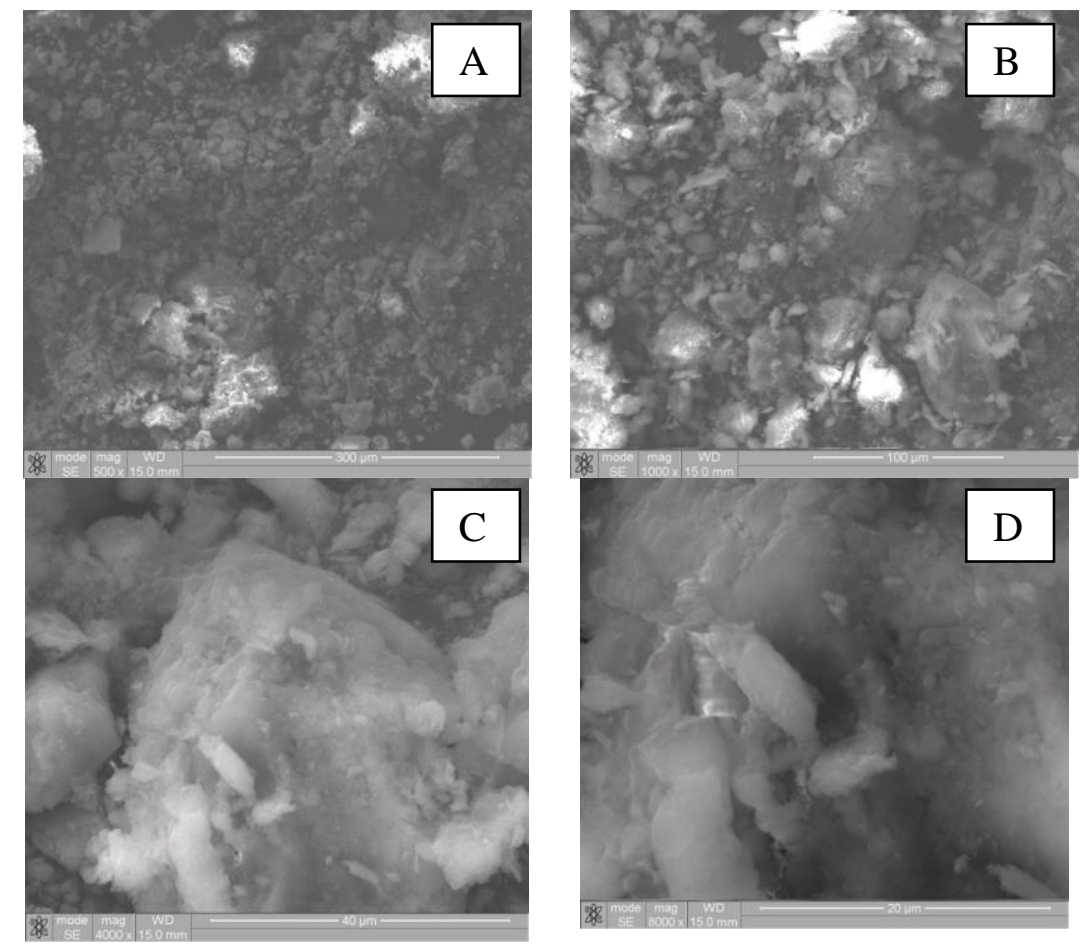

Fig. 3. Micrographs of montmorillonite clay. scale segment: a-300 microns, b- 100 microns, c- 40 microns, $\mathrm{d}-20$ microns

\section{Conclusion}

Currently, in Russia, research on the development of second-generation dosage forms transport drug systems, in which the main mechanism of transport is the sorption process, has not been conducted. The structural analysis of sorption substances allowed us to develop a classification of the possible interaction of the carrier substance with the drug molecule in the drug transport system.

According to the presented classification, the materials under study are divided into two groups according to their porous characteristics:

Group 1 - sorption interaction in pores and by ion exchange (Smectite, Montmorillonite Clay);

Group 2 - sorption in secondary pores and by means of oxygen and hydroxyl centers (Kaolin).

The perspective of further research is to model the porosity and sorption interaction of the carrier substance with the drug molecule in the drug transport system.

\section{References}

1. E. T. Zhilyakova, A. V. Bondarev, N. N. Bojko, D. K. Naplekov, Physical research of porosity of mineral sorbents, Journal of International Pharmaceutical Research, 45, (2018)

2. A. V. Bondarev, E. T. Zhilyakova, N. B. Demina, V. Y. Novikov, Study of Morphology of Sorption Substances, Drug development \& registration, 8 (2019) 
3. E. S. Novik, A. V. Dorenska, N. A. Borisova, O. V. Gunar, Estimation of the size and shape of particles of pharmaceutical substances by a microscopic method, Successes of Modern Science, 11 (2016) 